OPEN ACCESS

Edited by: Joseph O. Fadare, Ekiti State University,

Nigeria

Reviewed by:

Ludo Haazen,

Independent Researcher,

Mechelen, Belgium Kazeem Adeola Oshikoya,

Lagos State University,

Nigeria

*Correspondence:

Alfredo Vannacci

alfredo.vannacci@unifi.it

tThese authors share first authorship.

Specialty section: This article was submitted to Pharmaceutical Medicine and

Outcomes Research,

a section of the journal

Frontiers in Pharmacology

Received: 01 March 2019

Accepted: 24 July 2019

Published: 30 August 2019

Citation:

Lombardi N, Crescioli G, Bettiol A,

Tuccori M, Rossi M, Bonaiuti R,

Ravaldi C, Levi M, Mugelli A, Ricci S,

Lippi F, Azzari C, Bonanni $P$ and

Vannacci A (2019)

Vaccines Safety in Children and in General Population: A

Pharmacovigilance Study on Adverse

Events Following Anti-Infective

Vaccination in Italy.

Front. Pharmacol. 10:948.

doi: 10.3389/fphar.2019.00948

\section{Vaccines Safety in Children and in General Population: A Pharmacovigilance Study on Adverse Events Following Anti-Infective Vaccination in Italy}

\author{
Niccolò Lombardi it, Giada Crescioli ${ }^{1 \dagger}$, Alessandra Bettiol ${ }^{1}$, Marco Tuccori ${ }^{2,3}$, \\ Marco Rossi ${ }^{2,4}$, Roberto Bonaiuti ${ }^{1}$, Claudia Ravaldi ${ }^{5,6}$, Miriam Levi ${ }^{7}$, Alessandro Mugelli ${ }^{1}$, \\ Silvia Riccis,9, Francesca Lippi ${ }^{8,9}$, Chiara Azzari $6,8,9$, Paolo Bonanni ${ }^{6}$ and Alfredo Vannacci ${ }^{1,2,5 *}$ \\ ${ }^{1}$ Department of Neurosciences, Psychology, Drug Research and Child Health, Section of Pharmacology and Toxicology, \\ University of Florence, Florence, Italy, ${ }^{2}$ Tuscan Regional Centre of Pharmacovigilance, Florence, Italy, ${ }^{3}$ Unit of Adverse \\ Drug Reactions Monitoring, Department of Clinical and Experimental Medicine, University of Pisa, Pisa, Italy, ${ }^{4}$ Centre of \\ Pharmacovigilance, University Hospital of Siena, Department of Medical Sciences, Surgery and Neurosciences, University of \\ Siena, Siena, Italy, ${ }^{5}$ CiaoLapo, Charity for Healthy Pregnancy, Stillbirth and Perinatal Grief Support, Prato, Italy, ${ }^{6}$ Department \\ of Health Sciences, University of Florence, Florence, Italy, ${ }^{7}$ Epidemiology Unit, Department of Prevention, Local Health \\ Unit Tuscany Centre, Florence, Italy, ${ }^{8}$ Department of Pediatric Immunology, University of Florence, Florence, Italy, ${ }^{9}$ Meyer \\ Children's Hospital, Florence, Italy
}

Background: The concern for adverse events following immunization (AEFI) and antivaccination movements that lacked scientific evidence-based supports may reduce vaccine uptake in the general population. Thus, the aims of the present study were to characterize AEFI in general population (all age groups), in terms of frequency, preventability, and seriousness and to define predictors of their seriousness in children.

Methods: A retrospective study was performed on suspected AEFI reports for children and adults who received any form of vaccinations, collected in Tuscany, Italy, between 1 January and 31 December 2017. Patients' characteristics, suspected vaccines, and AEFI description were collected. Causality and preventability were assessed using $\mathrm{WHO}$ and Schumock and Thornton algorithms, respectively. Logistic regression was used to estimate the reporting odds ratios of potential predictors of AEFI seriousness in children.

Results: A total of 223 suspected AEFI reports were collected, and the majority of them were defined as non-serious (76.7\%). Reports were mostly related to one vaccine, and to a median of two to five strains/toxoids. The total number of simultaneously administered strains/toxoids and the presence of allergens did not correlate with AEFI seriousness. Considering vaccines with a high number of administered doses ( $\geq 60,000$ doses), the rates estimated for serious AEFI reports were always very low, ranging between 0.01 and 0.2/1,000 doses. Twenty-four vaccines (8,993 doses) were not related to any AEFI.

Conclusion: Results of present study showed that AEFI were very rare; the vast majority of them was non-serious and, despite the claims of anti-vaccination movements, the 
simultaneous administration of vaccines was safe and did not influence the risk of reporting a serious $\mathrm{AEFI}$, particularly in children.

Keywords: vaccines, adverse events following immunization, pharmacovigilance, safety, observational study, preventability, seriousness

\section{INTRODUCTION}

As vaccine-preventable infectious diseases continue to decline; parents and individuals in general have become increasingly concerned about the risks associated with vaccination. An adverse event following immunization (AEFI) is any untoward clinical occurrence which follows vaccinations and which does not necessarily have a causal relationship with vaccine use (Fulton et al., 2015; WHO, 2019a). An AEFI may be any unfavorable or unintended sign, abnormal laboratory finding, symptom, or disease. Moreover, AEFI classification may include: 1) vaccine product-related reaction (an AEFI that is caused or precipitated by a vaccine due to one or more of the inherent properties of the vaccine product), 2) vaccine quality defect-related reaction (an AEFI that is caused or precipitated by a vaccine that is due to one or more quality defects of the vaccine product including its administration device as provided by the manufacturer), 3) immunizationrelated errors, 4) immunization anxiety-related reactions, and 5) coincidental events (Fulton et al., 2015; WHO, 2019a). AEFI can be also represented by minor events, manifesting as local (i.e., erythema, edema, pain) or systemic (i.e., fever, exanthema, allergic reactions), and severe events manifesting as anaphylaxis, seizures, thrombocytopenia, hypotonic hyporesponsive episodes (HHE), or prolonged crying. Finally, an AEFI can be acute if it occurs within minutes after vaccine administration or delayed if it occurs several hours or days after vaccine administration (Danova et al., 2017). Considering the World Health Organization (WHO) criteria (WHO, 2019b), several considerations for assessing causality of an AEFI should be always evaluated: 1) temporal relationship, 2) alternative explanations, 3) proof of association, 4) prior evidence, 5) population-based evidence, and 6) biological plausibility. AEFI might be also related to strains/toxoids and/or to other vaccine components, such as: antibiotics (i.e., neomycin), components of the container (i.e., latex), residual proteins of the substrate (i.e., egg), or stabilizers (i.e., gelatine) (Autret-Leca et al., 2006).

In Italy, 18-25 AEFI reports are recorded every 100,000 vaccine doses. Overall, on a total of 4,766 AEFI reports collected during 2016 at the National level, more than $80 \%$ were defined as non-serious (AIFA, 2018). Nevertheless, in the last decade, public health authorities worldwide have observed an increase in the number of vaccine hesitant patients or parents (Larson et al., 2016) and an increase in "anti-vaccination" movements activities (Tafuri et al., 2015). The concern for vaccine safety and dearth of studies documenting surveillance of their adverse effects were the major theme of anti-vaccination movements
(Tafuri et al., 2015). As a consequence, immunization coverage in Italy has decreased both in children (Signorelli et al., 2017) and adults (Pezzotti et al., 2018) in recent years. Compulsory vaccination for children, therefore, became enacted into Law in July 2017 (Law n. 119) in order to address the vaccination short fall in Italy. Consequently, the number and uptake of compulsory vaccination have increased from 4 to 10 (Scavone et al., 2018b). These are those included in the hexavalent vaccine (diphtheria, tetanus, pertussis, poliomyelitis, hepatitis $\mathrm{B}$, and hemophilus influenzae $B$ ) and measles, mumps, rubella, and varicella, for children up to 6 years of age to be enrolled in kindergartens and pre-schools and for children up to 16 years of age to attend compulsory schools. Furthermore, health authorities are requested to promote all vaccines (mandatory and non-mandatory) recommended in the 2017-2019 National Immunisation Plan (Scavone et al., 2018b). As a matter of fact, vaccines such as those against Neisseria meningitidis, Streptococcus pneumoniae, or human papilloma virus are not included in the mandatory vaccination law; however, they are included in the recent Italian National Vaccination Plan (2015-2017) and are given free of charge to all newborns (Neisseria meningitidis and Streptococcus pneumoniae) or to all adolescents, both male and female (HPV vaccine).

Thus, improving AEFI characterization in the general and pediatric population remains a public health and a patient safety perception priority, especially after the new regulatory decision. Moreover, to the best of our knowledge, no studies have been previously conducted to assess the possible association between vaccines' characteristics (number of strains/toxoids simultaneously administered and presence of allergens as reported in the summary of product characteristic, SPC) and AEFI reporting.

The aim of the present study was to characterize AEFI in the general population evaluating their frequency in relation to the total number of administered doses, their preventability, and seriousness. Moreover, we also identified predictors of AEFI seriousness in children, by means of 1-year pharmacovigilance study.

\section{MATERIAL AND METHODS}

This is a retrospective study performed on reports of suspected AEFI collected in Tuscany, Italy, between 1 January and 31 December, 2017, and related only to vaccinations performed during such period. We analyzed all suspected AEFI reported from patients having at least one AEFI. All AEFI reports were collected through both the regional spontaneous reporting system and two active pharmacovigilance projects, Monitoring of the 
Adverse Effects in Pediatric population (MEAP) (Carnovale et al., 2014) and Epidemiological Monitoring of Adverse Drug Reactions in Emergency Department (MEREAFaPS) (Lombardi et al., 2018).

Each AEFI report demographic characteristics, including age, sex, and ethnic group, were recorded. Anatomical Therapeutic Chemical (ATC) classification system was used to code both vaccine administered and concomitant medications, for which we also recorded the administration route, therapy duration, and dosage. The description of AEFI according to diagnosis and symptoms was coded using the Medical Dictionary for Regulatory Activities (MedDRA) and organized by System Organ Class (SOC) (Lombardi et al., 2018). Seriousness of AEFI was classified according to the World Health Organization (WHO) criteria (Lombardi et al., 2018) as fatal, life-threatening, requiring hospitalization of the patient, or causing serious/ permanent disability.

The most frequently reported SOC and ATC classes were also evaluated. For each AEFI report, causality (categorized as consistent, inconsistent, indeterminate, or unclassifiable) and preventability (categorized as definitely or probably preventable, or not preventable) were assessed using the WHO algorithm (Tozzi et al., 2013; WHO, 2018) and Schumock and Thornton algorithm (Schumock and Thornton, 1992), respectively. Both assessments were performed by a multidisciplinary team composed by experts in clinical pharmacology, immunology, epidemiology, and pediatrics. The same team revised data on concomitant medications and analyzed vaccine-vaccine (VVI), vaccinedrug (VDI), and drug-drug (DDI) interactions using the drug interaction software Micromedex Drug-REAX System (Thomson Reuters Healthcare Inc., Greenwood Village, Colorado, United States), available online with restricted access (Santos et al., 2017). As reported in the Micromedex tool (Micromedex ${ }^{\circledR} 2.0$, 2013), interactions were classified as mild, moderate, or severe, depending on their clinical complexity.

For each vaccine, the number of bacterial or viral strains and toxoids was obtained from the specifications listed in the SPC. In case of multiple vaccines' administration in the same vaccination session, we obtained the total amount of strains/toxoids administered by summing the number of strains/toxoids for each product. Allergens listed in the SPC were considered together as a dichotomous variable (presence/absence). We considered the presence/absence of: neomycin and/or other antibiotics in traces, latex, egg, gelatine, etc.

For the general population (all age groups), the frequency of total and serious AEFI was calculated in relation to the total number of vaccines doses administered in Tuscany, Italy, in 2017. AEFI rates were calculated dividing the number of AEFI reports by the total number of administered doses for each ATC of interest and expressed as AEFI reports/administered doses $x$ 1,000. Data regarding AEFI reports and vaccines administered doses were obtained by the Regional Health Authority, after having been made anonymous. Due to the lack of information on total administered doses for influenza vaccines, all ATC classes related to these products were not included in the analysis, and AEFI rates for them were not calculated. AEFI reports potentially associated with those vaccines were nevertheless collected, assessed, and discussed.
For subjects $\leq 18$ years, predictors of AEFI seriousness, such as age groups, sex, number of vaccines simultaneously administered, vaccines characteristics (number of strains/toxoids and presence of allergens), and interactions (DDIs, VDIs, VVIs), were also evaluated. For children, age groups were codified using Food and Drug Administration (FDA) classification (FDA, 2014).

Descriptive statistics were used to summarize data. Categorical data were reported as frequencies and percentages and compared using the chi-square or Fischer exact tests, as appropriate, whereas continuous data were reported as median values with the related interquartile ranges (IQR) and compared using the Mann-Whitney test. Univariate and multivariate logistic regressions were used to estimate the reporting odds ratios (RORs) with $95 \%$ confidence intervals (CIs) of potential predictors of AEFI seriousness in children. ROR values for each variable were reported both as crude and adjusted values and adjusted for all the other considered variables. All results were considered to be statistically significant at $p<0.05$. Data management and statistical analysis were carried out using STATA 14.

The coordinating center of Tuscany, Italy, approved MEREAFaPS project (notification number 1225-December 21, 2009), and the local institutional ethics committee approved MEREAFaPS (study number 3055/2010, protocol number 45288-August 6, 2014) according to the legal requirements concerning observational studies. The MEAP project was approved in the frame of MEREAFaPS. Due to the retrospective nature of the present study and data anonymization, patient's consent to participate was not required.

\section{RESULTS}

In 2017, the entire population of Tuscany included around $3,736,968$ inhabitants, of which 601,160 (16\%) were children. Within the study period, a total of 223 AEFI reports were collected and evaluated; of them, 171 (76.7\%) were defined as non-serious, and $52(23.3 \%)$ were defined as serious. Table 1 shows the characteristics of all suspected AEFI reports (on the top of Table 1) and the characteristics of each AEFI (on the bottom of Table 1) in the general population.

\section{Characteristics of AEFI Reports}

The median age was 9.24 years (IQR, 1.14-42.17), and children $(\mathrm{n}=142,63.7 \%)$ experienced more AEFI than adults. However, there was no statistically significant difference among age groups when serious and non-serious AEFI reports were compared $(p=0.664)$. We encountered more AEFI reports referred to males than females, and the distribution of the type of events according to sex was significantly different $(\mathrm{p}=0.036)$. AEFI reports were more common among Caucasians.

Evaluating the causality assessment, 153 AEFI reports (68.6\%) were defined as consistent with the vaccination, $24(10.8 \%)$ as inconsistent, $44(19.7 \%)$ as indeterminate, and $2(0.9 \%)$ were defined as unclassifiable. Applying the Schumock and Thornton algorithm, more than $90 \%$ of suspected AEFI reports were classified as non-preventable, while 16 reports were defined as preventable. 
TABLE 1 | Characteristics of all AEFI reports (top) and each AEFI (bottom) in general population.

\begin{tabular}{|c|c|c|c|c|}
\hline & $\begin{array}{l}\text { Tot reports } \\
n=223(\%)\end{array}$ & $\begin{array}{c}\text { Non-serious } \\
n=171(\%)\end{array}$ & $\begin{array}{c}\text { Serious } \\
n=52(\%)\end{array}$ & p-Value \\
\hline \multicolumn{5}{|l|}{ N AEFI for each report } \\
\hline 1 & $76(34.08)$ & $57(33.33)$ & $19(36.54)$ & 0.503 \\
\hline 2 & $43(19.28)$ & $35(20.47)$ & $8(15.38)$ & \\
\hline 3 & $49(21.97)$ & $40(23.39)$ & $9(17.31)$ & \\
\hline $4+$ & $55(24.66)$ & $39(22.81)$ & $16(30.77)$ & \\
\hline \multicolumn{5}{|l|}{ Age, years (overall) } \\
\hline Median (IQR) & $9.24(1.14-42.17)$ & $9.95(1.24-44.25)$ & $2.26(0.84-22.28)$ & 0.091 \\
\hline \multicolumn{5}{|l|}{ Pediatric age groups (FDA classification) } \\
\hline Newborns (< 1 month) & 0 & 0 & 0 & 0.664 \\
\hline Infants (1 month-2 years) & $57(25.56)$ & $40(23.39)$ & $17(32.69)$ & \\
\hline Children (2-12 years) & $73(32.74)$ & $56(32.75)$ & 17 (32.69) & \\
\hline Adolescents (12-18 years) & $12(5.38)$ & $8(4.68)$ & $4(7.69)$ & \\
\hline \multicolumn{5}{|l|}{ Adults (quartiles of age) } \\
\hline 19-39 years & $20(8.97)$ & $16(9.36)$ & $4(7.69)$ & \\
\hline 40-49 years & $21(9.42)$ & $18(10.53)$ & $3(5.77)$ & \\
\hline $50-59$ years & $15(6.73)$ & $13(7.60)$ & $2(3.85)$ & \\
\hline Over 60 years & $25(11.21)$ & $20(11.70)$ & $5(9.62)$ & \\
\hline \multicolumn{5}{|l|}{ Sex } \\
\hline Female & $102(45.74)$ & $84(49.12)$ & $18(34.62)$ & 0.036 \\
\hline Male & $114(51.12)$ & $80(46.78)$ & $34(65.38)$ & \\
\hline Missing & $7(3.14)$ & 7 (4.09) & 0 & \\
\hline \multicolumn{5}{|l|}{ Ethnic group } \\
\hline Caucasian & $137(61.43)$ & $106(61.99)$ & $31(59.62)$ & 0.880 \\
\hline Others & $14(6.28)$ & $10(5.85)$ & $4(7.69)$ & \\
\hline Missing & $72(32.29)$ & $55(32.16)$ & $17(32.69)$ & \\
\hline \multicolumn{5}{|l|}{ Causality } \\
\hline Consistent & $153(68.61)$ & $129(75.44)$ & $24(46.15)$ & $<0.001$ \\
\hline Inconsistent & $24(10.76)$ & $7(4.09)$ & 17 (32.69) & \\
\hline Indeterminate & 44 (19.73) & $33(19.30)$ & $11(21.15)$ & \\
\hline Unclassifiable & $2(0.90)$ & $2(1.17)$ & 0 & \\
\hline \multicolumn{5}{|l|}{ Preventability } \\
\hline No & 207 (92.80) & $162(94.74)$ & $45(86.53)$ & 0.016 \\
\hline \multirow[t]{3}{*}{ Yes } & $16(7.20)$ & $9(5.26)$ & $7(13.47)$ & \\
\hline & Tot AEFI & Non-serious & Serious & p-Value \\
\hline & $n=570$ & $n=431$ & $n=139$ & \\
\hline \multicolumn{5}{|l|}{ Number of suspect drug for each AEFI } \\
\hline 1 & $489(85.79)$ & $379(87.94)$ & $110(79.14)$ & 0.002 \\
\hline 2 & $74(12.98)$ & $45(10.44)$ & $29(20.86)$ & \\
\hline 3 & $7(1.23)$ & $7(1.62)$ & 0 & \\
\hline \multicolumn{5}{|l|}{ Concomitant drugs (not suspected) } \\
\hline 0 & $502(88.07)$ & $383(88.86)$ & $119(85.61)$ & 0.023 \\
\hline 1 & $35(6.14)$ & $22(5.10)$ & $13(9.35)$ & \\
\hline 2 & $23(4.04)$ & $21(4.87)$ & $2(1.44)$ & \\
\hline 3 & $9(1.58)$ & $5(1.16)$ & $4(2.88)$ & \\
\hline 4 & $1(0.18)$ & 0 & $1(0.72)$ & \\
\hline \multicolumn{5}{|l|}{ Tot strains/toxoids } \\
\hline Median (IQR) & $4(4-7)$ & $4(3-7)$ & $4(4-6)$ & 0.815 \\
\hline 1 & $51(8.95)$ & $36(8.35)$ & $15(10.79)$ & 0.940 \\
\hline $2-5$ & $364(63.86)$ & $277(64.27)$ & $87(62.59)$ & \\
\hline $6-9$ & $53(9.30)$ & $40(9.28)$ & $13(9.35)$ & \\
\hline $10-13$ & $26(4.56)$ & $20(4.64)$ & $6(4.32)$ & \\
\hline $14+$ & $76(13.33)$ & $58(13.46)$ & $18(12.95)$ & \\
\hline \multicolumn{5}{|l|}{ Presence of allergens (in traces) } \\
\hline Yes & $222(38.95)$ & $180(41.76)$ & $42(30.22)$ & 0.015 \\
\hline No & $348(61.05)$ & $251(58.24)$ & $97(69.78)$ & \\
\hline \multicolumn{5}{|l|}{ Seriousness (out of 139) } \\
\hline Death & 0 & - & 0 & - \\
\hline Persistent or significant disability/incapacity & $8(5.76)$ & - & $8(5.76)$ & \\
\hline Congenital abnormalities/birth defects & 0 & - & 0 & \\
\hline Hospitalization or prolongation & $83(59.71)$ & - & $83(59.71)$ & \\
\hline Life-threatening condition & 0 & - & 0 & \\
\hline Other clinically relevant conditions & $48(34.53)$ & - & $48(34.53)$ & \\
\hline
\end{tabular}




\begin{tabular}{|c|c|c|c|c|}
\hline & $\begin{array}{l}\text { Tot reports } \\
n=223(\%)\end{array}$ & $\begin{array}{c}\text { Non-serious } \\
n=171(\%)\end{array}$ & $\begin{array}{c}\text { Serious } \\
n=52(\%)\end{array}$ & p-Value \\
\hline \multicolumn{5}{|l|}{ Outcome } \\
\hline Complete resolution & 247 (43.33) & $184(42.69)$ & 63 (45.32) & $<0.001$ \\
\hline Improvement & 119 (20.88) & $98(22.74)$ & $21(15.11)$ & \\
\hline Still unresolved\# & $78(13.68)$ & $50(11.60)$ & $28(20.14)$ & \\
\hline Resolution with sequelae & $5(0.88)$ & $5(1.16)$ & 0 & \\
\hline Unchanged/worsened reaction & $2(0.35)$ & $2(0.46)$ & 0 & \\
\hline Death & $5(0.88)$ & 0 & $5(3.60)$ & \\
\hline Missing $^{\circ}$ & $114(20.00)$ & $92(21.35)$ & $22(15.83)$ & \\
\hline \multicolumn{5}{|c|}{ Interactions (DDIs + VDls + VVIs) } \\
\hline Not applicable (one treatment) & 450 (78.95) & $346(80.28)$ & $104(74.82)$ & 0.032 \\
\hline No & $106(18.60)$ & $78(18.10)$ & $28(20.14)$ & \\
\hline Mild & 0 & 0 & 0 & \\
\hline Moderate & $2(0.35)$ & $2(0.46)$ & 0 & \\
\hline Severe & $12(2.11)$ & $5(1.16)$ & $7(5.04)$ & \\
\hline
\end{tabular}

IQR, interquartile range; FDA, Food and Drug Administration; DDI, drug-drug interaction; VDI, vaccine-drug interaction; WVI, vaccine-vaccine interaction.

\#From a case-by-case evaluation these events were classified as still unresolved due to the fact that the AEFI reporting was performed immediately after AEFI onset.

These events were classified as missing because the reporter did not complete the relative section present in the AEFI reporting form.

p-value was from Fisher Exact test for percentages and Mann-Whitney test for median values.

In particular, within preventable AEFI reports, we encountered: six cases of major VDIs observed between Pneumococcus, purified polysaccharides antigen-conjugated (ATC J07AL02), and acetaminophen; one case of moderate VDI observed between varicella, live-attenuated (ATC J07BK01), and betamethasone; one case of moderate VVI observed between Meningococcus $C$, purified polysaccharides antigen-conjugated (ATC J07AH07), and diphtheria-hemophilus influenzae B-pertussis-poliomyelitis-tetanushepatitis B (ATC J07CA09); three cases of post-immunization hypersensitivity reactions in patients with a previous history of allergy; three cases of patients who developed the same AEFI experienced following the administration of previous doses for the same vaccine (positive rechallenge); one case of therapeutic error; and one case of AEFI report observed following liveattenuated rotavirus (ATC J07BH01) administration in a patient with a previous history of intestinal intussusception.

\section{Characteristics of AEFI}

Regarding each AEFI $(n=570)$, the majority of them was associated with only 1 vaccine $(\mathrm{n}=489,85.8 \%)$, and $139(24.4 \%)$ were defined as "serious." Of the 139 serious AEFI reported in this study, $83(59.7 \%)$ resulted in hospitalization, and 48 were represented by "other clinically relevant conditions" (i.e., all adverse events judged by the reporter as clinically relevant because they required an intervention to prevent one of the other characteristics and consequences related to death, or life threatening, or hospitalization, or permanent disability, etc.). "Hospitalization or prolongation" group was mostly associated with vaccine-related fever, and within "other clinically relevant conditions" group; only two cases of death (for a total of five vaccine-AEFI pairs) were observed.

These fatal cases involved two women, aged 68 and 97 years old (data not shown in Table 1), who died for an acute respiratory tract infection (suspected vaccine ATC J07AL02) and for a cardiac failure in hypertensive cardiopathy (suspected vaccine ATC J07BB02), respectively. After an accurate clinical evaluation, these fatal cases were judged as not associated to vaccination. Finally, we encountered eight vaccine-AEFI pairs (derived from two AEFI reports) associated to "persistent or significant disability/incapacity" group, which were reported by patient/citizen and, consequently, misclassified. In fact, the clinical evaluation performed by the panel of experts revealed that these AEFI had indeed a complete resolution and were not causally consistent with vaccination.

Regarding the outcome, 366 (64.2\%) AEFI were completely resolved or improved, and $78(13.7 \%)$ were still unresolved at the time of reporting. The outcome was missing for 114 (20\%) AEFI. Finally, the outcome of five (0.9\%) AEFI was defined as resolution with sequelae and referred to a single patient for whom the observed events were not associated to the vaccination. Of note, as mentioned above, for a total of five vaccine-AEFI pairs $(0.9 \%)$ classified within the "other clinically relevant conditions" group and associated with the outcome death, the clinical evaluation revealed that these events were not causally correlated with vaccination.

Out of a total of 120 AEFI, only 14 of them presented a moderate $(n=2)$ or severe $(n=12)$ interaction. These interactionrelated AEFI derived from eight AEFI reports (seven cases of VDIs, one case of VVI) described above in the section of reports' characteristics.

Following MedDRA hierarchy, 267 (46.8\%) out of 570 AEFI belonged to "general disorders and administration site conditions," 67 (11.7\%) involved "nervous system disorders," $62(10.9 \%)$ involved "skin and subcutaneous tissue disorders," 60 (10.5\%) involved "gastrointestinal disorders," and 30 (5.3\%) involved "musculoskeletal disorders." Consequently, the five most frequently reported preferred terms were: fever $(17.5 \%)$, pain at site of vaccination $(5.3 \%)$, rash at site of vaccination $(5.3 \%)$, rash at unspecified site $(5.1 \%)$, and edema at site of vaccination (4.7\%) (Supplementary Table 1). Of note, within the SOC "nervous system disorders," only six cases of febrile-related seizures were reported, all of them with a complete resolution. 
Supplementary Table 2 shows the characteristics of all suspected AEFI reports (on the top) and the characteristics of each AEFI (on the bottom) observed for pediatric population, described taking into consideration the current National Vaccination Plan 2017-2019. As already reported for the general population, we observed similar results also for the pediatric one.

\section{Administered Doses and AEFI Reporting}

Considering all vaccines doses administered in Tuscany, we observed very few AEFI reports for vaccines with a high frequency of usage (more than 60,000 doses). In particular, as reported in Table 2, the most frequently administered vaccines were: Meningococcus B, multicomponent vaccine (117,525 doses); Meningococcus C, purified polysaccharides antigen-conjugated (109,515 doses); Pneumococcus, purified polysaccharides antigenconjugated (72,273 doses); diphtheria-hemophilus influenzae B-pertussis-poliomyelitis-tetanus-hepatitis $B \quad(65,158$ doses); and Meningococcus $A, C, Y$, and $W-135$, tetravalent purified polysaccharides antigen-conjugated (61,590 doses). For them, the rates of total and serious AEFI reports per 1,000 administered doses were very low and varied from 0.07 to 0.54 , and from 0.01 to 0.20 , respectively. Several vaccines were not associated with any AEFI. Supplementary Table 3 reports the number of administered doses of all ATC classes for which no AEFI reports were collected during the study period.

\section{Predictors of AEFI Seriousness in Children}

Table 3 shows demographic and clinical features associated with the risk of serious AEFI reporting in the pediatric age classes specifically mentioned in the National Vaccination Plan 2017-2019. Male sex and the presence of concomitant (not suspected) drugs were associated with a significantly higher risk of AEFI reported as serious. The total amount of strains/toxoids administered and the presence of allergens were not associated with AEFI seriousness, for all evaluated age groups.

\section{Distribution of AEFI Reports}

Figure 1 describes the distribution of AEFI reports collected for mandatory (J07CA09, J07CA02, J07BK01, J07BD54, J07BD52) and non-mandatory (J07BH01, J07AL02, J07AH09, J07AH07) vaccinations performed between 0 and 15 months of age, according to the National Vaccination Plan 2017-2019. For other vaccinations (mandatory and non-mandatory), no AEFI reports were collected in children aged 0-15 months. Dashed areas represents the age period when vaccination should be administered. The majority of AEFI reports (nonserious and serious) occurred within the time window for vaccine administration, demonstrating that vaccinations, were administered strictly following health authority dispositions. Some non-serious AEFI occurred before or after the recommended time window for vaccination, in particular to those related to Meningococcus B, multicomponent vaccine (J07AH09), and diphtheria-hemophilus influenzae B-pertussis-poliomyelitistetanus-hepatitis B (J07CA09). Data related to the other age groups are shown in the Supplementary Table 4.

\section{DISCUSSION}

This study aimed to characterize AEFI in the general population, evaluating their frequency in relation to the total number of administered doses and their preventability and seriousness, and to identify predictors of AEFI seriousness in children. To the best of our knowledge, this is the first study considering vaccine characteristics, in particular to the number of strains/toxoids simultaneously administered and the presence of allergens, as factors potentially associated to AEFI seriousness in patients aged $\leq 18$ years. Moreover, our study was performed in the light of the last Italian National Vaccination Plan 2017-2019.

In terms of immunization safety, our study showed a higher frequency of non-serious AEFI reports, both in the general and in the pediatric population. Moreover, the majority of the events were not preventable, highlighting the appropriateness of vaccines use and administration in the observed population. In fact, the specific algorithm used to assess AEFI preventability contains a section which helps the assessor to evaluate whether or not an AEFI is related to an inappropriate use of the vaccine (Schumock and Thornton, 1992). In particular, the answer "NO" to the points 2 and 3 (Section A) of the algorithm indicates a high level of appropriateness of vaccination. Most of the preventable AEFI reports were due to the potential interaction between the components of the vaccines or between the vaccines and concomitant medications administered to the patients. Previous studies have documented poor immunologic response to conjugated Pneumococcus vaccine following acetaminophen administration (Prymula et al., 2009; Falup-Pecurariu et al., 2017; Wysocki et al., 2017). We also reported one case of interaction between varicella, live-attenuated vaccine, and the glucocorticoid betamethasone, which may result in an inadequate immunological response to the live vaccine (Infectious Diseases and Immunization Committee, Canadian Paediatric Society, 2000; Liu et al., 2013). Finally, we collected a VVI reported between Meningococcus $C$, purified polysaccharides antigen-conjugated vaccine, and diphtheria-hemophilus influenzae B-pertussis-poliomyelitistetanus-hepatitis $B$ vaccine. The administration of Meningococcal groups $A, C$, and $W-135$ and $Y$ conjugate vaccine after the Tetanus toxoid vaccine may result in lower geometric mean titers against groups A, C, and W-135 (Rivera et al., 2018). In particular, the present interaction was observed in a 2 -year old female who received the two vaccinations out of the time window indicated for the administration of these vaccines, both in the SPC and in the National Vaccination Plan 2017-2019.

In our study, the most common AEFI related to vaccines were fever, injection-site reactions, rash, vomit, and fatigue, i.e., mild and transient untoward medical occurrences. Our results are comparable to those already published in literature, both in adults (Alguacil-Ramos et al., 2016; Mentzer et al., 2018) and in children (Danova et al., 2017; Lopes et al., 2018; Mentzer et al., 2018). We also reported six cases of febrile-related seizures (i.e., 1.05\% of AEFI). 
TABLE 2 | Number of administered doses and rates of AEFI reports in general population.

\begin{tabular}{|c|c|c|c|c|c|c|c|}
\hline Vaccine & ATC & $\begin{array}{l}\text { N strains/ } \\
\text { toxoids }\end{array}$ & $\begin{array}{c}\text { N AEFI } \\
\text { report } \\
\text { total }\end{array}$ & $\begin{array}{l}\text { N AEFI } \\
\text { report } \\
\text { serious }\end{array}$ & $\begin{array}{c}\mathbf{N} \\
\text { administered } \\
\text { doses }\end{array}$ & $\begin{array}{l}\text { Rate of total } \\
\text { AEFI reports } \\
\text { per } 1,000 \\
\text { doses }\end{array}$ & $\begin{array}{l}\text { Rate of serious } \\
\text { AEFI reports } \\
\text { per } 1,000 \\
\text { doses }\end{array}$ \\
\hline Meningococcus $\mathrm{B}$, multicomponent vaccine & J07AH09 & 4 & 63 & 23 & 117525 & 0.54 & 0.20 \\
\hline $\begin{array}{l}\text { Meningococcus } \mathrm{C} \text {, purified polysaccharides } \\
\text { antigen-conjugated }\end{array}$ & J07AH07 & 2 & 8 & 1 & 109515 & 0.07 & 0.01 \\
\hline $\begin{array}{l}\text { Pneumococcus, purified polysaccharides } \\
\text { antigen-conjugated }\end{array}$ & J07AL02 & 14 & 30 & 8 & 72273 & 0.42 & 0.11 \\
\hline $\begin{array}{l}\text { Diphtheria-hemophilus influenzae B-pertussis- } \\
\text { poliomyelitis-tetanus-hepatitis B }\end{array}$ & J07CA09 & 10 & 18 & 2 & 65158 & 0.28 & 0.03 \\
\hline $\begin{array}{l}\text { Meningococcus A, C, Y, and W-135 tetravalent } \\
\text { purified polysaccharides antigen-conjugated }\end{array}$ & J07AH08 & 5 & 27 & 2 & 61590 & 0.44 & 0.03 \\
\hline Pertussis, purified antigen, combinations with toxoids & J07AJ52 & 5 & 9 & 2 & 55478 & 0.16 & 0.04 \\
\hline $\begin{array}{l}\text { Measles, combinations with mumps, rubella and } \\
\text { varicella, live-attenuated }\end{array}$ & J07BD54 & 4 & 14 & 4 & 45989 & 0.30 & 0.09 \\
\hline Diphtheria-pertussis-poliomyelitis-tetanus & J07CA02 & 8 & 11 & 1 & 44188 & 0.25 & 0.02 \\
\hline Papillomavirus (human types 16,18 ) & J07BM02 & 4 & 4 & 0 & 21179 & 0.19 & 0.00 \\
\hline $\begin{array}{l}\text { Measles, combinations with mumps and rubella, } \\
\text { live-attenuated }\end{array}$ & J07BD52 & 3 & 23 & 8 & 18837 & 1.22 & 0.42 \\
\hline Tetanus toxoid, combinations with diphtheria toxoid & J07AM51 & 2 & 4 & 0 & 13598 & 0.29 & 0.00 \\
\hline Varicella, live-attenuated & J07BK01 & 1 & 4 & 1 & 13351 & 0.30 & 0.07 \\
\hline Tetanus toxoid & J07AM01 & 1 & 9 & 2 & 12669 & 0.71 & 0.16 \\
\hline Hepatitis B, purified antigen & J07BC01 & 1 & 6 & 1 & 8826 & 0.68 & 0.11 \\
\hline Hepatitis A, inactivated, whole virus & J07BC02 & 1 & 1 & 0 & 8803 & 0.11 & 0.00 \\
\hline Rota virus, live-attenuated & J07BH01 & 1 & 6 & 2 & 8499 & 0.71 & 0.24 \\
\hline Rota virus, pentavalent, live, reassorted & J07BH02 & 5 & 6 & 2 & 8499 & 0.71 & 0.24 \\
\hline Typhoid, oral, live-attenuated & J07AP01 & 1 & 2 & 1 & 3045 & 0.66 & 0.33 \\
\hline Poliomyelitis oral, trivalent, live-attenuated & J07BF02 & 3 & 1 & 0 & 2137 & 0.47 & 0.00 \\
\hline Poliomyelitis, trivalent, inactivated, whole virus & J07BF03 & 3 & & & & & \\
\hline Poliomyelitis oral, bivalent, live-attenuated & J07BF04 & 2 & & & & & \\
\hline Cholera, inactivated, whole cell & J07AE01 & 5 & 1 & 1 & 1495 & 0.67 & 0.67 \\
\hline $\begin{array}{l}\text { Papillomavirus (human types } 6,11,16,18,31,33 \text {, } \\
45,52,58 \text { ) }\end{array}$ & J07BM03 & 9 & 1 & 1 & 793 & 1.26 & 1.26 \\
\hline Rabies, inactivated, whole virus & J07BG01 & 1 & 1 & 0 & 439 & 2.28 & 0,00 \\
\hline Pneumococcus, purified polysaccharides antigen & J07AL01 & 23 & 2 & 1 & 351 & 5.70 & 2.85 \\
\hline $\begin{array}{l}\text { Diphtheria-hemophilus influenzae } \\
\text { B-pertussis-poliomyelitis-tetanus }\end{array}$ & J07CA06 & 8 & 1 & 1 & 152 & 6.58 & 6.58 \\
\hline
\end{tabular}

Although all of them were classified as serious, since they caused an emergency department (ED) admission, a complete resolution was reported in all cases. The majority of vaccines can cause fever, and some children who develop a fever can have a seizure. However, studies showed there is one case of fever-triggered seizure for every 3,000 to 4,000 children who receive a vaccine (Marin et al., 2010), and these seizures almost never cause harm over the long term (Committee to Review Adverse Effects of Vaccines, 2011). Considering AEFI-related ED visits or hospitalizations, the majority of them were referred to the preferred terms "fever" and "hyperpyrexia," adverse events well known and well characterized after vaccination (Sawyer et al., 2016).

Considering the high number of vaccine doses administered in Tuscany in 2017 (more than 600,000 doses), the total number of vaccine-related AEFI detected in the present study is low and provides a useful documentation of vaccination safety, both in adults and in children (Staltari et al., 2013). Notably, in terms of causality, no death was associated with vaccination. In fact, regarding the first case of decease, the suspected vaccine was ATC J07AL02 (Pneumococcus, purified polysaccharides antigen-conjugated), but clinical examination revealed that lung infection was attributable to a pneumococcal strain different from those contained in the 13 -valent vaccine, excluding a vaccination failure. In the second case of death, suspected vaccine was ATC J07BB02 (influenza, inactivated, split virus, or surface antigen), which caused a fever-related ED visit, but the decease occurred 15 days later and was related to a pre-existent heart failure.

Concurrent administration of vaccines with multiple strain/toxoid was not a predictor of serious AEFI in this study. Findings from previous studies are in agreement with our report (Shinefield et al., 1998; Miller et al., 2011; Vesikari et al., 2013) showing that two or more vaccines could be administered safely during the same vaccination session. Administering a child with several vaccines during the same visit offers some advantages, both in terms of an earlier onset of protection and in terms of saving costs and time. Moreover, the administration of multiple vaccines can represent a valuable measure to reduce pain at time of vaccination (Saleh et al., 2017). Babies' vaccination-related anxiety could be less significant when two or more vaccines are 
TABLE 3 | Association between serious AEFI risk and different factors expressed as reporting odds ratio (ROR) within pediatric population stratified according to the age classes of the National Vaccination Plan 2017-2019.

\begin{tabular}{|c|c|c|c|c|c|c|}
\hline & \multicolumn{2}{|c|}{ 0-15 months } & \multicolumn{2}{|c|}{16 months-12 years } & \multicolumn{2}{|c|}{$12-18$ years } \\
\hline & $\begin{array}{l}\text { Adjusted ROR } \\
\qquad(95 \% \mathrm{Cl})\end{array}$ & p-Value & $\begin{array}{l}\text { Adjusted ROR } \\
(95 \% \mathrm{Cl})\end{array}$ & p-Value & $\begin{array}{c}\text { Adjusted ROR } \\
(95 \% \mathrm{Cl})\end{array}$ & p-Value \\
\hline \multicolumn{7}{|l|}{ Sex } \\
\hline Female & Ref. & & & & & \\
\hline Male & $1.26(0.48-3.27)$ & 0.639 & $2.29(1.10-4.76)$ & 0.027 & $0.20(0.02-2.39)$ & 0.203 \\
\hline \multicolumn{7}{|c|}{$\begin{array}{l}\text { Concomitant drugs } \\
\text { (not suspected) }\end{array}$} \\
\hline No & Ref. & & & & & \\
\hline Yes & $3.20(0.96-10.70)$ & 0.059 & $6.88(1.42-33.43)$ & 0.017 & - & - \\
\hline \multicolumn{7}{|c|}{ Tot strains/toxoids } \\
\hline $1-6$ & Ref. & & & & & \\
\hline $6+$ & $0.16(0.02-1.30)$ & 0.087 & $2.14(0.97-4.74)$ & 0.060 & - & - \\
\hline \multicolumn{7}{|c|}{$\begin{array}{l}\text { Presence of } \\
\text { allergens (in traces) }\end{array}$} \\
\hline No & Ref. & & & & & \\
\hline Yes & $0.31(0.04-2.56)$ & 0.280 & $0.94(0.45-1.98)$ & 0.873 & - & - \\
\hline
\end{tabular}

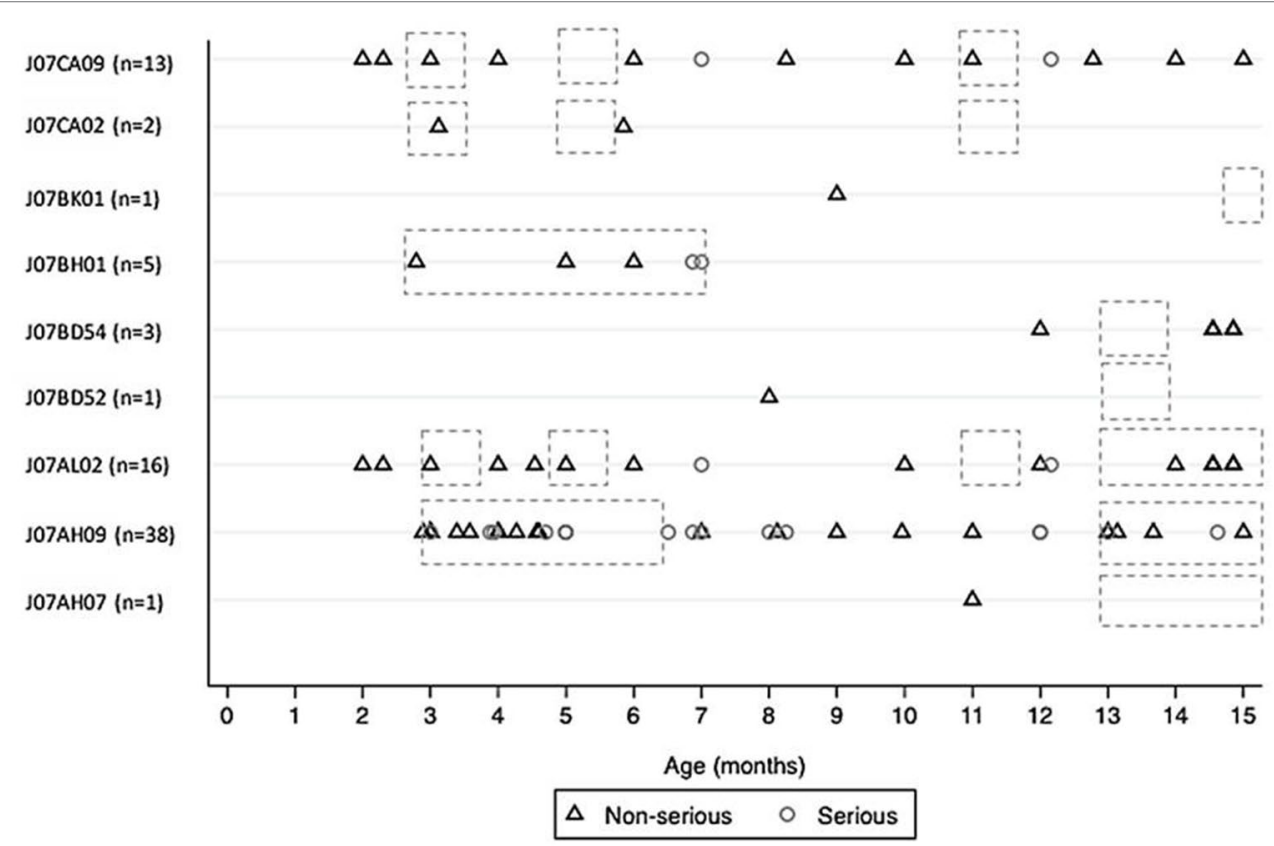

FIGURE 1 | Distribution of AEFI reports (non-serious and serious) collected for mandatory and non-mandatory vaccinations performed between 0 and 15 months of age, according to the National Vaccination Plan 2017-2019 (dashed areas). Mandatory: "Diphtheria-hemophilus influenzae B-pertussis-poliomyelitis-tetanushepatitis B" (J07CA09); "Diphtheria-pertussis-poliomyelitis-tetanus" (J07CA02); "varicella, live-attenuated" (J07BK01); "measles, combinations with mumps, rubella and varicella, live-attenuated" (J07BD54); "measles, combinations with mumps and rubella, live-attenuated" (J07BD52). Non-mandatory: "Rotavirus, live-attenuated" (J07BH01); "Pneumococcus, purified polysaccharides antigen-conjugated” (J07AL02); "Meningococcus B, multicomponent vaccine” (J07AH09); "Meningococcus C, purified polysaccharides antigen-conjugated" (J07AH07).

administered once than in more than one vaccination sessions (Dodd, 2003). Reducing the number of sessions provides benefits not only to infants, but also to parents, healthcare providers, office managers, and managed care administrators.

In terms of AEFI, our results are comparable with those published in the scientific literature over the past decades, both in adults (Alguacil-Ramos et al., 2016; Mentzer et al., 2018) and in children (Danova et al., 2017; Lopes et al., 2018; Mentzer et al., 2018). The adverse events related to the use of vaccines occur at a very low frequency and are generally irrelevant when compared to risks associated to non-vaccination (Aps et al., 2018). The overall literature provides no substantial indication of a causal relationship between immunization and the very occasional serious AEFI (Swedish Council on Health Technology Assessment, 2009). According to a 2014 study from the Centers for Disease Control and Prevention (CDC) (Whitney et al., 2014), 
vaccines have saved thousands of lives worldwide, in particular in children, preventing more than 300 million kids from getting sick only in United States. CDC also reported that the majority of AEFI (more than 90\%) described in literature are generally not serious. A 2011 report from the National Academy of Medicine reviewed more than 1,000 vaccine studies and concluded that serious AEFI to vaccines are rare (Committee to Review Adverse Effects of Vaccines, 2011).

Another concern about vaccination safety refers to the presence of allergens, but as reported in our study, allergens could be considered safe and harmless (Matsumoto et al., 2017). To date, only few allergens in traces are currently present in human vaccines, and available evidences have already demonstrated their safety (Bastola et al., 2017). Data from the present study confirm that the presence of allergens in vaccines is not correlated with the onset of AEFI.

Focusing on Italy, the 2018 annual report on post-marketing surveillance of vaccines published by the Italian Medicines Agency (AIFA) showed a national rate of AEFI reporting of 11.1 per 100,000 inhabitants for all vaccines (mandatory and nonmandatory) (AIFA, 2018), while for Tuscany alone is of 8.0 per 100,000 inhabitants. According to our data, the actual rate for Tuscany is even lower (5.95 per 100,000 inhabitants). In fact, in our analysis, only AEFI reports associated with vaccinations performed during 2017 were evaluated. Instead, AIFA annual report contains also AEFI reports referred to vaccination administered several years before 2017. The other variables considered by AIFA in the annual report (i.e., sex, age, SOCs, preferred terms, etc.) are consistent with our results.

\section{STRENGTHS AND LIMITATIONS}

This study has several limitations and strengths. First, its retrospective nature may have led to an underestimation of AEFI rate, since not all patients presenting an AEFI, even if serious, attend ED or spontaneously report the adverse event. However, considering that reporting all observed AEFI is compulsory for health professionals, and furthermore, data on AEFI reports in Tuscany were collected both through the regional spontaneous reporting system and two active pharmacovigilance projects, the issue of underreporting can be considered of relatively low relevance. Second, our analysis is based on AEFI reports that are affected by limits that include inaccurate and incomplete information, mainly related to lack of clinical data (Scavone et al., 2018a). Given that, we cannot always exclude the absence of information that was not listed in AEFI reports, and that might have influenced the clinical evaluation of each report (i.e., the lack of vaccination's date or previous/current patient medical conditions which could affect the evaluation of causality assessment). Moreover, we have only Tuscan AEFI reports, which cannot be considered fully representative of the other Italian regions and, although our cases were all clinically evaluated, due to the large number of statistical tests performed the outcome of these tests should be regarded as descriptive. Given that the information on influenza vaccines administration is recorded in an administrative database different from those used for our analysis, we were not able to calculate the rate of AEFI to influenza vaccines in our sample.

Despite these limitations, this is the first retrospective analysis performed in Italy on potential predictors of AEFI seriousness in children over 1 year of observation. Moreover, focusing on AEFI reporting rate, since we evaluated AEFI related only to vaccination performed during 2017, our rate can be considered less influenced by the number of AEFI reports derived from the mass media influence on vaccination hesitancy observed in Italy before Law 119/2017 (introducing mandatory vaccination for 10 vaccines) was approved. We strongly believe that pharmacovigilance studies are a valid scientific tool, simple and inexpensive, allowing healthcare professionals to detect and better characterize AEFI in clinical practice. In fact, we were able to highlight vaccination safety, showing a very low rate of AEFI and demonstrating that, particularly in children, their seriousness is not related to the number of vaccines and strains/toxoids simultaneously administered.

\section{CONCLUSION}

AEFI is a major concern, both regarding the public opinion as well as the healthcare politics, and it represents a challenge for healthcare professional and healthcare services. The present study provided new insights on the factors that might influence the risk of AEFI reporting in children (i.e., concomitant drugs, number of strains/toxoids simultaneously administered, and presence/ absence of allergens). Based on our results, the risks associated with immunization do not justify vaccination avoidance, especially because, among the risks related to vaccines, non-vaccination could be considered the most important. In this context, novel and specific educational approaches are needed in order to transmit the importance to correctly understand scientific research on vaccine safety to the younger generations of citizens, who will be the parents of tomorrow.

\section{DATA AVAILABILITY}

Datasets are available on request.

\section{AUTHOR CONTRIBUTIONS}

Study design was contributed by NL, GC, AB, and AV, with assistance from the rest of the authors. $\mathrm{AB}$ took the lead in data analysis, assisted by NL and GC, and data interpretation was performed by $\mathrm{NL}, \mathrm{GC}, \mathrm{AV}, \mathrm{CA}$, and PB, with assistance from the other authors. The manuscript was written primarily by NL and GC, with assistance from the other authors, and revised by MT, MR, RB, CR, ML, AM, $\mathrm{SR}$, and FL. All authors approved the final version of the manuscript.

\section{FUNDING}

This study was funded by a research grant from the AIFA (the Italian Medicines Agency), Rome, Italy, Tuscan County resolution DGRT 790/2016 All. C. The funder of the study had 
no role in the collection, analysis and interpretation of data, nor in the writing of the report, nor in the decision to submit the article for publication.

\section{ACKNOWLEDGMENTS}

The Authors would like to thank all health-care professionals (clinicians, nurses, hospital pharmacists, and pharmacy students)

\section{REFERENCES}

AIFA. (2018). Italian Vaccines Report 2017. Available at: http://www.aifa.gov. it/sites/default/files/Rapporto_Vaccini_2017_vers._acc.pdf. Last accessed: June 18, 2019.

Alguacil-Ramos, A. M., Muelas-Tirado, J., Garrigues-Pelufo, T. M., Portero-Alonso, A., Diez-Domingo, J., Pastor-Villalba, E., et al. (2016). Surveillance for adverse events following immunization (AEFI) for 7 years using a computerised vaccination system. Public Health 135, 66-74. doi: 10.1016/j. puhe.2015.11.010

Aps, L., Piantola, M. A. F., Pereira, S. A., Castro, J. T., Santos, F. A. O., and Ferreira, L. C. S. (2018). Adverse events of vaccines and the consequences of non-vaccination: a critical review. Rev. Saude Publica 52, 40. doi: 10.11606/ S1518-8787.2018052000384

Autret-Leca, E., Bensouda-Grimaldi, L., Jonville-Bera, A. P., and Beau-Salinas, F. (2006). [Pharmacovigilance of vaccines]. Arch. Pediatr. 13, 175-180. doi: 10.1016/j.arcped.2005.10.020

Bastola, R., Noh, G., Keum, T., Bashyal, S., Seo, J. E., Choi, J., et al. (2017). Vaccine adjuvants: smart components to boost the immune system. Arch. Pharm. Res. 40, 1238-1248. doi: 10.1007/s12272-017-0969-Z

Carnovale, C., Brusadelli, T., Zuccotti, G., Beretta, S., Sullo, M. G., Capuano, A., et al. (2014). The importance of monitoring adverse drug reactions in pediatric patients: the results of a national surveillance program in Italy. Expert Opin. Drug Saf. 13 Suppl 1, S1-S8. doi: 10.1517/14740338.2014.928692

Committee to Review Adverse Effects of Vaccines (2011). Adverse effects of vaccines: evidence and causality, eds. K. Stratton, A. Ford, E. Rusch \& E.W. Clayton. (Washington, DC).

Danova, J., Kocourkova, A., and Celko, A. M. (2017). Active surveillance study of adverse events following immunisation of children in the Czech Republic. BMC Public Health 17, 167. doi: 10.1186/s12889-017-4083-4

Dodd, D. (2003). Benefits of combination vaccines: effective vaccination on a simplified schedule. Am. J. Manag. Care 9, S6-12.

Falup-Pecurariu, O., Man, S. C., Neamtu, M. L., Chicin, G., Baciu, G., Pitic, C., et al. (2017). Effects of prophylactic ibuprofen and paracetamol administration on the immunogenicity and reactogenicity of the 10 -valent pneumococcal nontypeable Haemophilus influenzae protein D conjugated vaccine (PHiD-CV) co-administered with DTPa-combined vaccines in children: an open-label, randomized, controlled, non-inferiority trial. Hum. Vaccin. Immunother. 13, 649-660. doi: 10.1080/21645515.2016.1223001

Fda (2014). Pediatric exclusivity study age group. Available at: https://www.fda. gov/drugs/data-standards-manual-monographs/pediatric-exclusivity-studyage-group. Last accessed: June 19, 2019.

Fulton, T. R., Narayanan, D., Bonhoeffer, J., Ortiz, J. R., Lambach, P., and Omer, S. B. (2015). A systematic review of adverse events following immunization during pregnancy and the newborn period. Vaccine 33, 6453-6465. doi: 10.1016/j. vaccine.2015.08.043

Infectious Diseases and Immunization Committee, Canadian Paediatric Society. (2000). A guide to contraindications to childhood vaccinations. Can. J. Infect. Dis. 11, 13-14

Larson, H. J., De Figueiredo, A., Xiahong, Z., Schulz, W. S., Verger, P., Johnston, I. G., et al. (2016). The state of vaccine confidence 2016: global insights through a 67-country survey. E. Bio. Med. 12, 295-301. doi: 10.1016/j. ebiom.2016.08.042

Liu, D., Ahmet, A., Ward, L., Krishnamoorthy, P., Mandelcorn, E. D., Leigh, R., et al. (2013). A practical guide to the monitoring and management of the participating to the MEREAFaPS and MEAP studies, in particular Dr. Emanuela Balocchini, Dr. Roberto Banfi, and Dr. Maria Parrilli.

\section{SUPPLEMENTARY MATERIAL}

The Supplementary Material for this article can be found online at: https://www.frontiersin.org/articles/10.3389/fphar.2019.00948/ full\#supplementary-material

complications of systemic corticosteroid therapy. Allergy Asthma Clin Immunol. 9, 30. doi: 10.1186/1710-1492-9-30

Lombardi, N., Crescioli, G., Bettiol, A., Marconi, E., Vitiello, A., Bonaiuti, R., et al. (2018). Characterization of serious adverse drug reactions as cause of emergency department visit in children: a 5-years active pharmacovigilance study. BMC Pharmacol. Toxicol. 19, 16. doi: 10.1186/s40360-018-0207-4

Lopes, S. R. C., Perin, J. L. R., Prass, T. S., Carvalho, S. M. D., Lessa, S. C., and Dorea, J. G. (2018). Adverse events following immunization in Brazil: age of child and vaccine-associated risk analysis using logistic regression. Int. J. Environ. Res. Public Health 15 (6), E1149. doi: 10.3390/ijerph15061149

Marin, M., Broder, K. R., Temte, J. L., Snider, D. E., Seward, J. F. Centers for Disease Control, et al. (2010). Use of combination measles, mumps, rubella, and varicella vaccine: recommendations of the Advisory Committee on Immunization Practices (ACIP). MMWR Recomm. Rep. 59, 1-12.

Matsumoto, M., Komatsu, S. I., Ikeda, T., Shimomura, K., Watanabe, K., Hirabayashi, K., et al. (2017). Investigating toxicity specific to adjuvanted vaccines. Regul. Toxicol. Pharmacol. 91, 29-38. doi: 10.1016/j.yrtph.2017.09.003

Mentzer, D., Oberle, D., and Keller-Stanislawski, B. (2018). Adverse events following immunisation with a meningococcal serogroup B vaccine: report from post-marketing surveillance, Germany, 2013 to 2016. Euro. Surveill. 23, (17). doi: 10.2807/1560-7917.ES.2018.23.17.17-00468

Micromedex $^{\circledR}$ 2.0. (2013). Medication, disease and toxicology management. Available 1093 at: http://www.micromedexsolutions.com/home/dispatch. Last accessed: 1094 September 06, 2018.

Miller, E., Andrews, N., Waight, P., Findlow, H., Ashton, L., England, A., et al. (2011). Safety and immunogenicity of coadministering a combined meningococcal serogroup $\mathrm{C}$ and Haemophilus influenzae type b conjugate vaccine with 7-valent pneumococcal conjugate vaccine and measles, mumps, and rubella vaccine at 12 months of age. Clin. Vaccine Immunol. 18, 367-372. doi: 10.1128/CVI.00516-10

Pezzotti, P., Bellino, S., Prestinaci, F., Iacchini, S., Lucaroni, F., Camoni, L., et al. (2018). The impact of immunization programs on 10 vaccine preventable diseases in Italy: 1900-2015. Vaccine 36, 1435-1443. doi: 10.1016/j. vaccine.2018.01.065

Prymula, R., Siegrist, C. A., Chlibek, R., Zemlickova, H., Vackova, M., Smetana, J., et al. (2009). Effect of prophylactic paracetamol administration at time of vaccination on febrile reactions and antibody responses in children: two open-label, randomised controlled trials. Lancet 374, 1339-1350. doi: 10.1016/S0140-6736(09)61208-3

Rivera, L., Schwarz, T. F., Kim, K. H., Kim, Y. K., Behre, U., Cha, S. H., et al. (2018). Immunogenicity and safety of the quadrivalent meningococcal vaccine MenACWY-TT co-administered with a combined diphtheria-tetanus-acellular pertussis vaccine versus their separate administration in adolescents and young adults: a phase III, randomized study. Vaccine 36, 4750-4758. doi: 10.1016/j. vaccine.2018.04.034

Saleh, E., Swamy, G. K., Moody, M. A., and Walter, E. B. (2017). Parental Approach to the prevention and management of fever and pain following childhood immunizations: a survey study. Clin. Pediatr. (Phila) 56, 435-442. doi: $10.1177 / 0009922816675116$

Santos, T. R. A., Silveira, E. A., Pereira, L. V., Provin, M. P., Lima, D. M., and Amaral, R. G. (2017). Potential drug-drug interactions in older adults: a population-based study. Geriatr. Gerontol. Int. 17, 2336-2346. doi: 10.1111/ggi.13070

Sawyer, M. H., Simon, G., and Byington, C. (2016). Vaccines and febrile seizures: quantifying the risk. Pediatrics 138 (1), e20160976. doi: 10.1542/peds.2016-0976 Scavone, C., Rafaniello, C., Brusco, S., Bertini, M., Menditto, E., Orlando, V., et al. (2018a). Did the new Italian law on mandatory vaccines affect adverse event 
following immunization's reporting? A pharmacovigilance study in Southern Italy. Front. Pharmacol. 9, 1003. doi: 10.3389/fphar.2018.01003

Scavone, C., Sessa, M., Clementi, E., Rossi, F., and Capuano, A. (2018b). Italian immunization goals: a political or scientific heated debate? Front. Pharmacol. 9, 574. doi: 10.3389/fphar.2018.00574

Schumock, G. T., and Thornton, J. P. (1992). Focusing on the preventability of adverse drug reactions. Hosp. Pharm. 27, 538.

Shinefield, H. R., Black, S. B., Staehle, B. O., Adelman, T., Ensor, K., Ngai, A., et al. (1998). Safety, tolerability and immunogenicity of concomitant injections in separate locations of M-M-R II, VARIVAX and TETRAMUNE in healthy children vs. concomitant injections of M-M-R II and TETRAMUNE followed six weeks later by VARIVAX. Pediatr. Infect. Dis. J. 17, 980-985. doi: 10.1097/00006454-199811000-00003

Signorelli, C., Odone, A., Cella, P., Iannazzo, S., D’ancona, F., and Guerra, R. (2017). Infant immunization coverage in Italy (2000-2016). Ann. Ist. Super. Sanita. 53, 231-237. doi: 10.4415/ANN_17_03_09

Staltari, O., Cilurzo, F., Caroleo, B., Greco, A., Corasaniti, F., Genovesi, M. A., et al. (2013). Annual report on adverse events related with vaccines use in Calabria (Italy): 2012. J. Pharmacol. Pharmacother. 4, S61-S65. doi: 10.4103/0976-500X.120951

Stratton K, Ford A, Rusch E, Clayton. EW, editors. (2011). Adverse Effect of vaccines evidence and causality. Washington (DC): The National Academies Press.

Swedish Council on Health Technology Assessment. (2009). Vaccines to children: protective effect and adverse events: a systematic review. Stockholm: SBU Yellow Report No. 191.

Tafuri, S., Gallone, M. S., Calabrese, G., and Germinario, C. (2015). Adverse events following immunization: is this time for the use of WHO causality assessment? Expert Rev. Vaccines 14, 625-627. doi: 10.1586/14760584.2015.1029460

Tozzi, A. E., Asturias, E. J., Balakrishnan, M. R., Halsey, N. A., Law, B., and Zuber, P. L. (2013). Assessment of causality of individual adverse events following immunization (AEFI): a WHO tool for global use. Vaccine 31, 5041-5046. doi: 10.1016/j.vaccine.2013.08.087

Vesikari, T., Esposito, S., Prymula, R., Ypma, E., Kohl, I., Toneatto, D., et al. (2013). Immunogenicity and safety of an investigational multicomponent, recombinant, meningococcal serogroup B vaccine (4CMenB) administered concomitantly with routine infant and child vaccinations: results of two randomised trials. Lancet 381, 825-835. doi: 10.1016/S0140-6736(12)61961-8 Whitney, C. G., Zhou, F., Singleton, J., Schuchat, A., and Centers for Disease, C., and Prevention (2014). Benefits from immunization during the vaccines for children program era - United States, 1994-2013. MMWR Morb. Mortal. Wkly. Rep. 63, 352-355.

WHO. (2018). Causality assessment of an adverse event following immunization (AEFI). Available at: http://apps.who.int/iris/bitstream/handle/10665/259959/ 9789241513654-eng.pdf;jsessionid=C958C729EDA0D32661FB1986193025F2? sequence $=1$. Last accessed: September 06, 2018.

WHO. (2019a). Adverseeventsfollowing immunization (AEFI). Availableat:https:// www.who.int/vaccine_safety/initiative/detection/AEFI/en/. Last accessed: June 18, 2019.

WHO. (2019b).Vaccine safety basics. Available at: http://vaccine-safety-training. org/frequency-and-severity.html. Last accessed: September 06, 2018.

Wysocki, J., Center, K. J., Brzostek, J., Majda-Stanislawska, E., Szymanski, H., Szenborn, L., et al. (2017). A randomized study of fever prophylaxis and the immunogenicity of routine pediatric vaccinations. Vaccine 35, 1926-1935. doi: 10.1016/j.vaccine.2017.02.035.

Conflicts of Interest Statement: The authors declare that the research was conducted in the absence of any commercial or financial relationships that could be construed as a potential conflict of interest.

Copyright (๑ 2019 Lombardi, Crescioli, Bettiol, Tuccori, Rossi, Bonaiuti, Ravaldi, Levi, Mugelli, Ricci, Lippi, Azzari, Bonanni and Vannacci. This is an open-access article distributed under the terms of the Creative Commons Attribution License (CC BY). The use, distribution or reproduction in other forums is permitted, provided the original author(s) and the copyright owner(s) are credited and that the original publication in this journal is cited, in accordance with accepted academic practice. No use, distribution or reproduction is permitted which does not comply with these terms. 